\title{
Réaction des éleveurs bovins des régions semi-arides algériennes face aux contraintes économiques et climatiques
}

\author{
Lounis Semara ${ }^{1,2, *}$, Toufik Madani ${ }^{1}$, Charefeddine Mouffok ${ }^{1}$ et Farida Belkasmi ${ }^{1,3}$ \\ ${ }^{1}$ Département des sciences agronomiques, université Ferhat Abbes, Sétif 01, Algérie \\ 2 Centre universitaire Abedelhafid Boussouf, Mila, Algérie \\ ${ }^{3}$ Université Mohamed El Bachir El Ibrahimi, Bordj Bou Arréridj, Algérie
}

\begin{abstract}
Résumé - Les évolutions socioéconomiques récentes de l'Algérie et l'accentuation de l'aridité du climat local induisent des transformations dans les pratiques et les stratégies des exploitations agricoles. Cette étude analyse la réaction des petites exploitations familiales d'élevage bovin des régions semi-arides de Sétif face aux contraintes économiques et aux incertitudes du milieu. L'approche retenue est l'identification des trajectoires d'évolution des exploitations agricoles d'élevage bovin sur une période de cinq ans, entre 2012 et 2017. L'analyse montre que les exploitations suivies ont développé six types de trajectoires d'adaptation pour maintenir leur production et répondre aux pressions externes et internes aux exploitations. Généralement, on observe une réduction des effectifs du cheptel bovin et des surfaces consacrées aux fourrages, ainsi qu'une chute de la proportion d'exploitations spécialisées en élevage bovin et en production laitière. Cette étude montre l'importance d'intégrer la dynamique des exploitations d'élevage dans les programmes régionaux de développement agricole.
\end{abstract}

Mots clés : adaptation de la production / contrainte climatique / agriculture familiale / système d'élevage / bovin

\begin{abstract}
Reaction of cattle farmers in Algerian semi-arid regions to economic and climatic constraints. The recent socioeconomic changes in Algeria and the accentuation of the aridity of the local climate induce transformations in farming practices and strategies. This study analyses the adaptation strategies implemented by small family cattle farms in the Setif semi-arid region, to face economic uncertainties and environmental constraints. The approach used is the identification of evolution trajectories of cattle farms over a period of five years, between 2012 and 2017. The analysis shows that the farms have developed six types of adaptation trajectories to maintain their production and respond to external and internal pressures. There is a decrease in the size of the herd and in areas dedicated to cultivated fodder, as well as a reduction in the proportion of farms specialized in cattle and milk production. This study shows the importance of integrating the dynamics of livestock farming into regional agricultural development programs.
\end{abstract}

Keywords: adjustment of production / climatic constraint / family farming / rearing systems / cattle

\section{Introduction}

Les agriculteurs développent des stratégies d'adaptation face aux incertitudes auxquelles ils sont confrontés (Ingrand et al., 2009). Ces incertitudes concernent la survenance d'événements climatiques extrêmes, la variation des prix et l'inadaptation des politiques publiques (Lherm et al., 2004). Pour les agriculteurs, plus particulièrement pour les éleveurs d'herbivores, la recherche de nouvelles cohérences dans le fonctionnement de leurs exploitations nécessite de prendre ces

\footnotetext{
* Auteur de correspondance : lounisemara@gmail.com
}

éléments en compte (Cochet et Devienne, 2005). L'exploration des adaptations que les agriculteurs apportent à leurs systèmes techniques de production constitue une base importante dans la recherche des moyens pour concilier, au niveau de l'exploitation et selon les spécificités du milieu, les objectifs de durabilité de l'élevage (Gibon et al., 2011).

Dans le cas des exploitations agricoles de la région semiaride algérienne, l'évolution de la situation socioéconomique du pays a eu des effets directs sur le secteur agricole, caractérisé à l'origine par une fragilité des systèmes de production (Abbas et al., 2002). Parmi ces effets, on peut citer l'inflation du prix des intrants agricoles importés, notamment des aliments concentrés du bétail, et les difficultés 


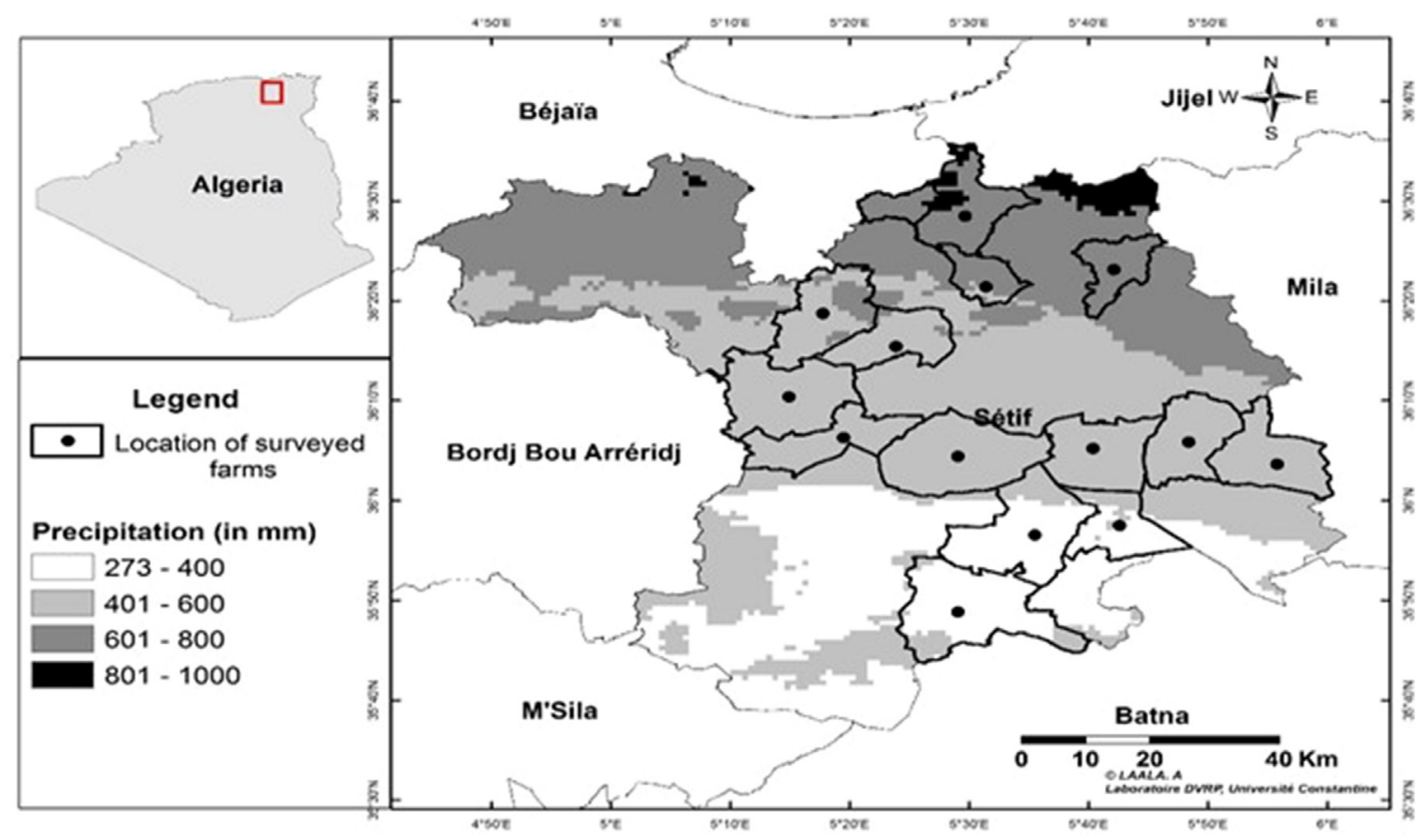

Fig. 1. Région d'étude et localisation des exploitations étudiées.

Fig. 1. Study area and location of surveyed farms.

d'encaissement des primes pour les produits agricoles soutenus par l'État, tels que le lait. À cela s'ajoute l'influence de conditions climatiques locales marquées, notamment ces dernières années, par un déficit et une irrégularité des précipitations et de longs épisodes de sécheresse. Selon les données météorologiques de la région de Sétif, l'écart en termes de pluviométrie annuelle moyenne est de presque $100 \mathrm{~mm}$ entre $2012(384 \mathrm{~mm})$ et 2016 $(288 \mathrm{~mm})$. L'étude de Rouabhi et al. (2018) confirme ces évolutions marquantes du climat de la région.

Dans cette conjoncture, cette étude s'intéresse aux petites exploitations familiales intégrant un atelier bovin. Elle a pour objectif le diagnostic des modes d'adaptation mis en place à moyen terme (entre 2012 et 2017) par les agroéleveurs de la région de Sétif en région semi-aride algérienne. S'interroger sur la dynamique des exploitations d'élevage et analyser leurs chemins d'évolution permet de préciser les arrangements techniques et agroéconomiques proposés en réponse aux pressions économiques et climatiques. À travers une description des modalités de réaction des décideurs dans ces exploitations, on cherche à comprendre l'ampleur des ajustements effectués sur les systèmes de production face aux perturbations, pour identifier les sources et les clés de sécurisation du processus de production. Cette démarche permettra de définir le rôle qui peut être octroyé à la présence d'un cheptel bovin dans des systèmes de production à faible niveau de structuration et dans un environnement incertain, élément qui semble crucial pour concevoir l'avenir des exploitations régionales et de la filière bovine.

\section{Matériels et méthodes}

\subsection{Région d'étude}

La région de Sétif fait partie de la région semi-aride algérienne. Elle est située à une altitude moyenne de $1080 \mathrm{~m}$ et se caractérise par un climat continental semi-aride avec trois étages bioclimatiques. La moyenne des précipitations annuelles est d'environ $400 \mathrm{~mm}$ pour l'ensemble de la wilaya (Fig. 1). Les températures dépassent souvent les $40^{\circ} \mathrm{C}$ en été et descendent en dessous de zéro en hiver.

\subsection{Collecte des données}

Les exploitations agricoles, objets de cette étude, sont de type familial. Les données ont été collectées en deux étapes lors d'enquêtes de terrains directives auprès des agroéleveurs de la région de Sétif. La première enquête a été réalisée en 2012 ; une base de données relative à 310 agriculteurs intégrant un élevage bovin a alors été construite (le critère de choix des éleveurs était l'élevage de deux vaches laitières au minimum). Cet échantillon a fait l'objet d'une étude non publiée sur la diversité des systèmes de production et d'élevage dans la région. Un échantillon restreint de 55 producteurs-éleveurs extrait du premier échantillon a été interrogé une seconde fois en 2017. Les exploitations de la seconde enquête ont été choisies sur la base des conclusions de la première enquête, de telle façon que ces exploitations représentent la diversité agricole de la région (Fig. 1).

\subsection{Analyse des données}

Après une description des évolutions caractérisant les structures des exploitations et des cheptels, ainsi que les niveaux de spécialisation (en élevage, en élevage bovin spécifiquement, et en production lait ou viande), une typologie des trajectoires d'évolution des exploitations a été construite. Dans un premier temps, 15 variables permettant de décrire les changements ayant eu lieu au cours de la période 2012-2017 ont été choisies. Douze variables présentent l'ampleur de l'évolution des structures 
Tableau 1. Évolution des structures agricoles et des niveaux de spécialisation.

Table 1. Evolution of farm structure and specialization levels.

\begin{tabular}{|c|c|c|}
\hline Paramètres structurels & $\begin{array}{l}\text { Situation } 2012 \\
\text { (moyenne } \pm \text { écart-type) }\end{array}$ & $\begin{array}{l}\text { Situation } 2017 \\
\text { (moyenne } \pm \text { écart-type) }\end{array}$ \\
\hline Surface agricole exploitée (ha) & $20,1 \pm 22,0$ & $18,3 \pm 14,5$ \\
\hline Surface céréalière (blé + orge) (ha) & $09,7 \pm 8,8$ & $11,1 \pm 10,4$ \\
\hline $\begin{array}{l}\text { Surface fourragère (prairie permanente }+ \\
\text { fourrages cultivés) (ha) }\end{array}$ & $07,4 \pm 15,7$ & $04,5 \pm 3,6$ \\
\hline Taille du cheptel ruminant (UGB) & $23,7 \pm 17,4$ & $17,9 \pm 12,8$ \\
\hline Taille du cheptel bovin (UGB) & $17,8 \pm 11,9$ & $14,9 \pm 11,9$ \\
\hline Cheptel bovin/cheptel ruminant & $75 \%$ & $83 \%$ \\
\hline Taille du cheptel ovin (UGB) & $05,9 \pm 13,7$ & $03,0 \pm 4,4$ \\
\hline Taille du cheptel vaches laitières & $10,7 \pm 6,6$ & $07,8 \pm 7,6$ \\
\hline Vaches laitières/cheptel bovin & $60 \%$ & $52 \%$ \\
\hline \multicolumn{3}{|l|}{ Spécialisation en élevage } \\
\hline Élevage & $14,5 \%$ & $17,5 \%$ \\
\hline Élevage-céréales & $65,5 \%$ & $69,5 \%$ \\
\hline Élevage-polyculture & $20 \%$ & $13 \%$ \\
\hline \multicolumn{3}{|l|}{ Spécialisation en élevage bovin } \\
\hline Bovins & $70,4 \%$ & $47,8 \%$ \\
\hline Bovins-ovins & $18,5 \%$ & $43,5 \%$ \\
\hline Bovins-aviculture & $5,5 \%$ & $6,5 \%$ \\
\hline Bovins-élevage diversifié & $5,6 \%$ & $2,2 \%$ \\
\hline \multicolumn{3}{|l|}{ Spécialisation en production lait ou viande } \\
\hline Lait & $12,7 \%$ & $6,5 \%$ \\
\hline
\end{tabular}

UGB : unité gros bétail.

agricoles sous forme de modalités qualitatives (Tab. 2) et trois variables illustrent l'objectif de changement des niveaux de spécialisation (Fig. 3). Cette méthodologie a été partiellement adaptée de travaux d'autres chercheurs (Hostiou, 2003 ; Dockès et al., 2013). Dans un second temps, des trajectoires-types ont été mises en scène à l'aide d'une analyse des correspondances multiples suivie d'une classification de type cluster hiérarchique. L'étude des déterminants du changement à l'échelle de l'exploitation a été effectuée par une projection des centroïdes des trajectoires sur les caractéristiques structurelles de l'exploitation et sur le profil de l'exploitant (Fig. 4); elle a été réalisée en utilisant un tracé des catégories avec CATPCA (categorical principal components analysis) dans IBM SPSS 24.

\section{Résultats}

\section{1 Évolution des structures des exploitations et des niveaux de spécialisation}

Les résultats du tableau 1 montrent qu'en moyenne les surfaces emblavées en céréales ont augmenté. Cette pratique s'est répercutée négativement sur la sole fourragère ( -3 ha en moyenne par exploitation en 2017 par rapport à 2012) et sur celle réservée au pâturage. Cela explique qu'il y ait eu une réduction de la taille du cheptel de ruminants. Le cheptel bovin a diminué, et notamment le cheptel de vaches laitières ( -3 vaches en moyenne par exploitation en 2017 par rapport à 2012). L'examen des changements ayant affecté les niveaux de spécialisation montre qu'entre 2012 et 2017, le nombre d'éleveurs spécialisés dans l'élevage bovin a diminué (70\% en 2012 contre $48 \%$ en 2017 ), alors que le nombre de producteurs détenant des cheptels bovins et ovins a nettement augmenté ( $18 \%$ en 2012 contre $43 \%$ en 2017).

\subsection{Arrêt définitif de l'activité agricole et d'élevage: dans quelles situations?}

Dans l'échantillon étudié, neuf exploitations agricoles (environ $16 \%$ des exploitations) ont cessé définitivement leurs activités agricoles. Ces exploitations de vaches laitières avaient été créées grâce aux dispositifs étatiques de lutte 
Tableau 2. Intensité du changement dans les structures agricoles, par trajectoire.

Table 2. Intensity of change in farm structure, by trajectory.

\begin{tabular}{|c|c|c|c|c|c|c|}
\hline Paramètres structurels & T1 & $\mathbf{T 2}$ & T3 & $\mathbf{T 4}$ & T5 & T6 \\
\hline Changement de surface agricole exploitée & ++ & - & $=$ & - & - & ++ \\
\hline Changement de surface céréalière (blé + orge) & ++ & -- & $=$ & $=$ & $=$ & + \\
\hline $\begin{array}{l}\text { Changement de surface fourragère (prairie permanente }+ \\
\text { fourrages cultivés) }\end{array}$ & + & + & - & - & $=$ & - \\
\hline Changement de surface de jachère pâturée & $=$ & + & - & - & $=$ & $=$ \\
\hline Changement de taille du cheptel de ruminants & ++ & ++ & $=$ & - & -- & --- \\
\hline Changement de taille du cheptel bovin & ++ & + & $=$ & - & --- & $=$ \\
\hline Changement de taille du cheptel ovin & $=$ & + & $=$ & $=$ & ++ & --- \\
\hline Changement du rapport cheptel bovin/cheptel élevé & $=$ & -- & $=$ & $=$ & -- & ++ . \\
\hline Changement de taille du cheptel de vaches laitières & + & $=$ & - & - & --- & - \\
\hline $\begin{array}{l}\text { Changement de taille du cheptel bovin viande (taurillons }+ \\
\text { génisses viande }+ \text { bœufs d'engrais }+ \text { vaches de réforme) }\end{array}$ & + & + & + & - & -- & $=$ \\
\hline Changement du rapport vaches laitières/cheptel bovin & - & - & -- & $=$ & $=$ & $=$ \\
\hline Changement du rapport bovins viande/cheptel bovin & + & + & ++ & $=$ & $=$ & $=$ \\
\hline
\end{tabular}

$\mathrm{T}$ : trajectoire ; intensité du changement observé : + à $+++:$ augmentation; $=:$ stabilité $;$ - à - - - : réduction. Les cellules grisées représentent les changements qui caractérisent le plus la trajectoire.

contre le chômage. Les propriétaires étaient des jeunes sans expérience en élevage et en agriculture, ayant débuté leurs activités avec des systèmes fragiles (hors-sol). Ces systèmes sont les plus vulnérables aux mutations économiques et climatiques. Les raisons de la disparition de ces exploitations sont multiples: augmentation des prix des fourrages et de l'aliment concentré, difficultés liées aux procédures administratives d'encaissement de la prime laitière et difficultés pour payer les prêts contractés auprès des banques.

\subsection{Diversité des trajectoires pour maintenir la production}

Six types de trajectoires ont été identifiés sur l'échantillon des 46 exploitations ayant maintenu leur production (Fig. 2). Les facteurs les plus discriminants des trajectoires d'évolution sont le redimensionnement de la taille du cheptel bovin et l'amplitude des changements effectués sur les niveaux de spécialisation en élevage. Les principales caractéristiques de ces trajectoires sont résumées dans le tableau 2 et la figure 3.

\subsubsection{Trajectoire 1-Être «gros » en bovins et en céréales (8 exploitations)}

À l'origine, les producteurs de cette catégorie sont des éleveurs bovins; leur plan d'action consiste à accroître leur effectif bovin ( +13 unités gros bétail [UGB]/exploitation) et leur surface agricole exploitée en céréales $(+8$ ha/exploitation) sans modifier les niveaux de spécialisation de leur élevage. Une partie d'entre eux (3 exploitations) optent pour une diversification des activités agricoles (élevage-céréales au lieu de l'élevage seul) et une stabilité dans le système de production bovin (scénario T01-B dans la figure 3), alors que les autres (5 exploitations) cherchent une diversification des produits d'origine bovine (lait et engraissement), alors qu'auparavant, ils étaient spécialisés en production laitière.

\subsubsection{Trajectoire 2-Investir dans la diversité de l'élevage (5 exploitations)}

Les pratiques mises en place consistent à diversifier les productions animales (mise en place d'un nouvel atelier ovin de 7 UGB) et à augmenter la taille du cheptel bovin viande principalement (+6 UGB/exploitation). La localisation de ces exploitations dans un territoire plus affecté par l'aridité a conduit les exploitants à réduire les surfaces des céréales $(-4 \mathrm{ha} /$ exploitation) pour libérer davantage de parcours pour les ovins.

\subsubsection{Trajectoire 3-S'adapter techniquement via le cheptel bovin et sa production (12 exploitations)}

Pour ce groupe d'exploitations, la source de flexibilité est la réorientation de la production bovine. Les éleveurs ou céréaliers-éleveurs s'adaptent en changeant la structure du cheptel bovin: remplacer des vaches laitières $(-3$ vaches/ exploitation) par des taurillons d'engraissement $(+2 \mathrm{UGB} /$ exploitation), ou l'inverse. La moitié des exploitations (6 exploitations) dans cette trajectoire (T03-A dans la figure 3) ont gardé un système de production stable, mais ont agi sur la structure du cheptel bovin en augmentant l'effectif de taurillons d'engraissement (+3UGB/exploitation). Les changements techniques, pour des exploitants auparavant spécialisés en production laitière, consistent à aller vers des systèmes de production mixtes lait-engraissement en modifiant le rapport vaches laitières-bovins viande. Pour trois autres exploitations (scénario T03-B dans la figure 3), la stratégie est inverse: stabilité de l'effectif de vaches laitières et élimination de l'atelier d'engraissement.

3.3.4 Trajectoire 4-Rester "stable », mais ajuster la taille du troupeau bovin (10 exploitations)

Ce groupe rassemble les exploitations qui sont restées stables dans leur niveau de spécialisation en élevage bovin, mais ont opté pour une réduction de la taille du cheptel bovin 


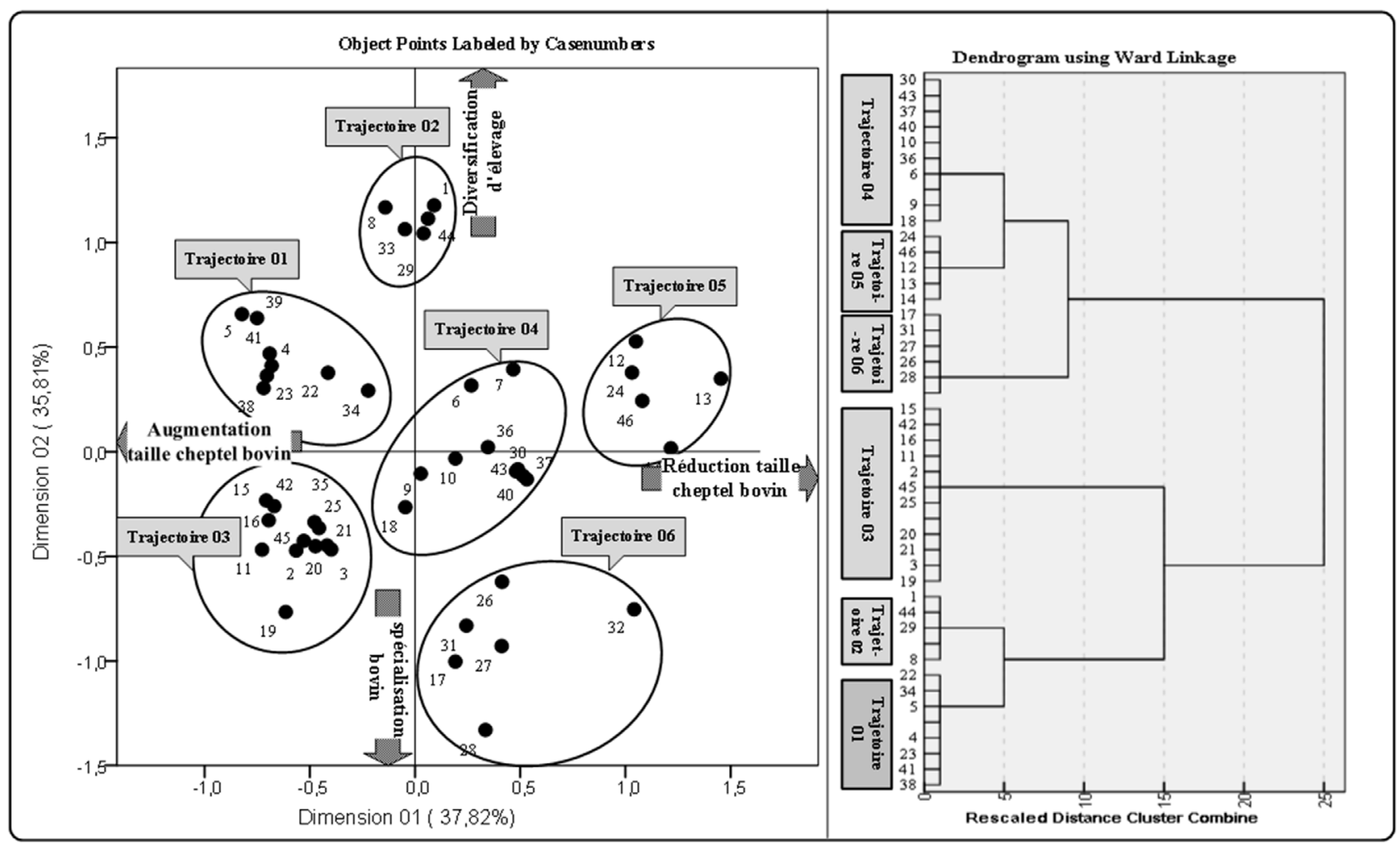

Fig. 2. Carte factorielle et dendrogramme de la typologie des trajectoires d'évolution des exploitations.

Fig. 2. Factorial map and dendrogram of the typology of farm evolution trajectories.

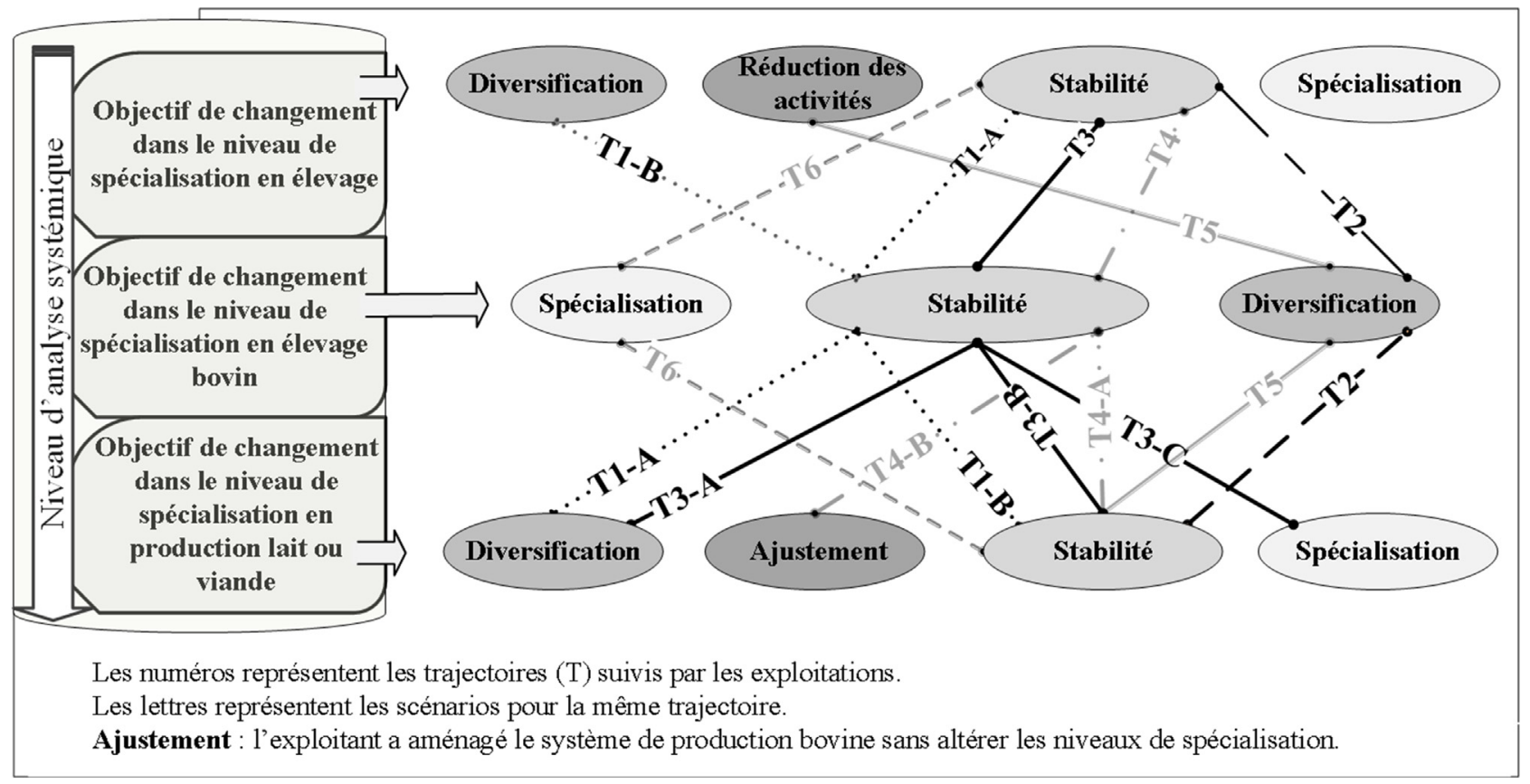

Fig. 3. Objectifs des changements dans les niveaux de spécialisation, par trajectoire.

Fig. 3. Objectives of changes in specialization levels, by trajectory. 


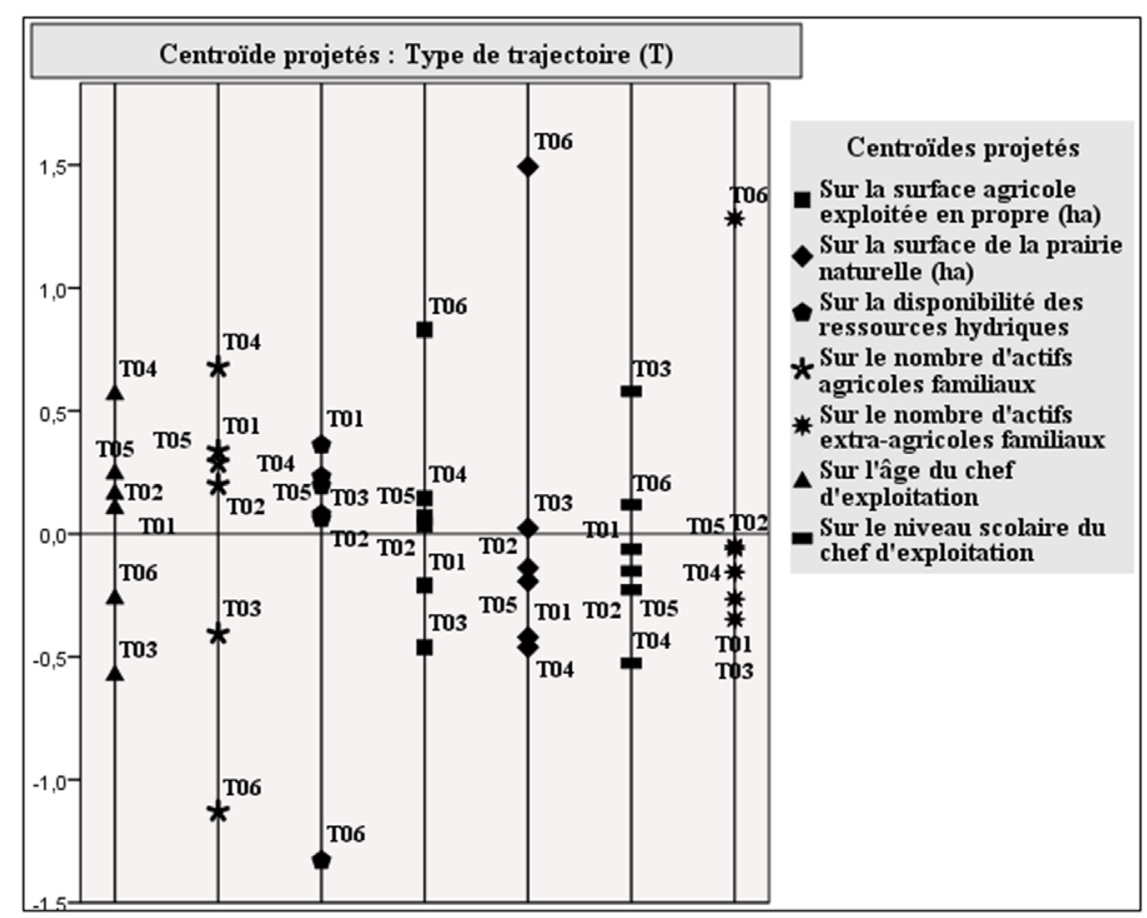

Fig. 4. Tracé des trajectoires projetées sur les déterminants de production.

Fig. 4. Plot of trajectories projected on production determinants.

(-9 UGB/exploitation). On note une diminution sensible du nombre de vaches laitières ( -5 vaches/exploitation) en réponse à un manque d'offre en fourrages.

\subsubsection{Trajectoire 5-Maîtriser les aléas via un élevage moins gros et non spécialisé (5 exploitations)}

Dans cette trajectoire, les éleveurs ont réorganisé leur système pour être plus opérationnels en élevage. Ils ont réduit le nombre d'activités agricoles pratiquées, passant d'un système élevage-agriculture diversifiée (céréaliculture et maraîchage) à un système élevage-céréales, afin de développer la possibilité de varier les productions animales. Ils vont vers l'association bovins-ovins (+6 UGB ovin/exploitation) au lieu d'un élevage spécialisé en bovins. Une autre façon pour maîtriser les aléas est de réduire considérablement le nombre de têtes de bovins ( $-28 \mathrm{UGB} /$ exploitation), les vaches laitières étant la cible principale de ces actions ( -14 vaches/exploitation).

\subsubsection{Trajectoire 6-Maintenir les bovins tant qu'il y a des ressources fourragères (6 exploitations)}

Cette trajectoire se caractérise par des réductions sensibles de l'effectif de ruminants élevés ( $-34 \mathrm{UGB} /$ exploitation). La restructuration du troupeau consiste à diminuer le nombre de bovins $(-2 \mathrm{UGB})$, mais surtout à éliminer le troupeau ovin (-32 UGB/exploitation). Cette trajectoire semble plus imposée que réellement choisie. L'élimination des ovins et le maintien d'un atelier bovin s'expliquent par le fait que ces exploitations disposent de prairies naturelles. Les niveaux de spécialisation en élevage et en production bovine lait-viande sont maintenus stables, mais les surfaces en céréales sont élargies (+3 ha/exploitation). Cela constitue une stratégie de sécurisation du système de production animale, fragilisé par la spécialisation bovine.

\subsection{Déterminants du changement à l'échelle de l'exploitation}

L'examen des facteurs supposés déterminants des choix effectués par les agroéleveurs de la région de Sétif (Fig. 4) a montré que la trajectoire 1 est favorisée par la disponibilité de la main-d'œuvre agricole familiale et les ressources en eau. En effet, les membres actifs n'exercent aucune activité extraagricole. La disponibilité des ressources en eau (forages, oueds et retenues collinaires) est un atout stratégique de développement des cultures fourragères en irrigué (notamment l'avoine en vert), favorisant l'augmentation de la taille du cheptel bovin. Dans le cas des trajectoires 2 et 5 , il semble que le choix soit indépendant des facteurs intra-exploitation évoqués dans la figure 4, sachant que ces deux stratégies se caractérisent par un même changement. La trajectoire 3 répond au profil de jeunes exploitants ayant un niveau de scolarisation élevé, qui travaillent sur des surfaces agricoles très restreintes. Les choix dont ils disposent en termes de conversion sont limités. Pour eux, la façon adéquate de s'adapter est de modifier la composition du troupeau bovin et le type de produit. Les opportunités de diversification des activités agricoles ou l'augmentation de la taille du cheptel nécessitent plus de ressources et imposent des charges supplémentaires en matière de main-d'œuvre. À l'inverse, les exploitations de la trajectoire 4, qui se caractérisent par une certaine stabilité dans les 
niveaux de spécialisation, sont gérées par des chefs âgés et au niveau d'instruction faible. Ces éleveurs sont moins motivés à changer leurs orientations, étant donné qu'ils sont en fin de carrière professionnelle. Ils perçoivent moins que les autres les changements de leur environnement et répondent moins rapidement aux pressions externes. La trajectoire 6 est favorisée par les disponibilités foncières et les ressources naturelles. L'élimination du troupeau ovin dans ces exploitations s'explique par le manque de disponibilité en maind'œuvre familiale, résultat de l'abandon de l'agriculture par les membres de ces familles qui cherchent des activités extraagricoles.

\section{Discussion}

\subsection{Des structures fragiles engendrent des systèmes non stables}

Cette étude montre que, globalement, une des réactions marquantes des agroéleveurs de la région est le retour vers un élevage diversifié (bovin-ovin notamment). La diversification des troupeaux est une stratégie qui permet de faire face à l'incertitude (Tichit et al., 2004). De nombreuses recherches montrent les avantages des systèmes mixtes cultures-élevage en matière de durabilité économique par rapport aux activités agricoles spécialisées (Ryschawy et al., 2013). Les éleveurs de bovins qui ont vécu l'expérience de la spécialisation laitière reviennent vers des systèmes mixtes, moins sensibles aux aléas. Plusieurs facteurs peuvent être à l'origine de ces changements, principalement la non-rentabilité économique $\mathrm{du}$ lait comme produit principal, notamment après l'inflation du prix des aliments concentrés. En France, dans l'étude de Rapey (2016), il ressort que certaines formes de conversion sont survenues essentiellement après la crise laitière de 20082009. La sécheresse chronique qui a sévi en Algérie durant la période 2012-2017 (plus de $100 \mathrm{~mm} /$ an de déficit par rapport à la moyenne de pluviométrie annuelle dans la région) s'est répercutée négativement sur l'autonomie fourragère des élevages, alors que celle-ci est une clef de voûte de la viabilité économique et de la pérennité des exploitations d'élevage face aux incertitudes du climat, des politiques publiques et des marchés (Belland, 2011).

\subsection{Changer pour s'adapter: une décision complexe dépendant de plusieurs facteurs}

Dans le cas des élevages bovins soumis à des contraintes hydriques et foncières, les pratiques communes des producteurs sont la décapitalisation du cheptel (notamment le cheptel bovin) et le changement du niveau de spécialisation de l'élevage. Ces constatations sont similaires à celles de Begon et al. (2009) dans la région du Ségala dans le Massif central français. Les facteurs dont dépendent les choix des agroéleveurs sont multiples: principalement le foncier agricole, les ressources naturelles en fourrages et particulièrement la maind'œuvre agricole familiale. Dans l'étude de Sraïri et al. (2018), les exploitations ayant le plus de disponibilités en terres et en capital (exploitations sous la trajectoire 6 dans notre cas) peuvent choisir de garder le bétail et de l'associer avec des céréales. Benniou (2008) a montré que, dans les cas où il est possible de mobiliser de l'eau d'irrigation et de pratiquer l'élevage bovin, le dynamisme de l'agriculteur peut conduire à une certaine diversification des systèmes de production. Le profil du chef d'exploitation est déterminant dans notre situation. En effet, le chef d'exploitation établit ses choix et développe ses stratégies en fonction de son histoire personnelle, de son âge et de sa situation familiale, de son niveau de formation et de ses compétences (Rouget, 2008).

\section{Conclusion}

La caractérisation de l'évolution des exploitations d'élevage bovin dans la région de Sétif en Algérie sous la pression des contraintes économiques et climatiques, a permis de mettre en évidence des changements profonds dans les structures d'exploitation et des cheptels. Les réactions des agroéleveurs ont conduit à des formes d'organisation différentes qui dépendent des spécificités de l'exploitation. L'étude a permis d'identifier six types de réactions. Une partie des producteurs renforcent leur système à travers la capitalisation d'un grand cheptel, d'autres à travers la diversification des productions. Les exploitations fragiles réduisent considérablement la taille du troupeau et changent la composition du cheptel. La stabilité de l'exploitation n'est qu'apparente, les éleveurs réagissent de plusieurs façons différentes (diminuer le cheptel en année défavorable, modifier l'orientation productive du troupeau bovin). Si le choix de la diversification apparaît comme un pilier de la durabilité économique de l'exploitation face aux perturbations extérieures, la spécialisation pourrait être davantage le résultat d'une conjugaison de contraintes intraet extra-exploitation. Les projets de développement local de la filière bovine doivent prendre en considération ces constats, car l'efficacité des actions à mettre en place dépend de l'intégration de la diversité des structures des exploitations agricoles, des différents projets des éleveurs et de la dynamique des systèmes de production, ainsi que de leurs capacités à faire face aux contraintes.

\section{Références}

Abbas K, Madani T, Ben Cheick EH, Merouche L. 2002. Systèmes d'élevage ovin en zone semi-aride céréalière : taille d'exploitation et caractère pastoral. New Medit 1: 50-55.

Begon M, Pailleux JY, Joly N, Lemery B, Dedieu B. 2009. Les chemins pour durer en élevage bovin laitier : diversité des logiques d'action sur le long terme en Ségala (Massif central). Renc Rech Ruminants 16: 105-108.

Belland C. 2011. Étude de l'influence des marchés et des politiques publiques agricoles sur la dynamique des systèmes de polycultureélevage dans les Coteaux de Gascogne. Master Pro ERG Agrocampus-Ouest, $68 \mathrm{p}$.

Benniou R. 2008. Les systèmes de production dans les milieux semiarides en Algérie: analyse agronomique de leur diversité et des systèmes de culture céréaliers dans les hautes plaines sétifiènnes. Thèse de doctorat, Alger: INA, 293p.

Cochet H, Devienne S. 2005. Comprendre l'agriculture en région agricole: question de méthode sur l'analyse en termes de systèmes de production. Paris: INAPG, UER agriculture comparée et développement agricole, $18 \mathrm{p}$.

Dockès AC, Boisdon I, Experton C, Fourdin S. 2013. Mise au point de méthodes et outils innovants pour développer l'élevage laitier 
biologique en zones de piémont et montagne. Innovations agronomiques 32: 243-257.

Gibon A, Ryschawy J, Schaller N, Blouet A, Coquil X, Martin P, et al. 2011. L'élevage, un atout pour le développement durable des territoires dans les régions de polyculture-élevage. Renc Rech Ruminants 18: 369-372.

Hostiou N. 2003. Pratiques et stratégies de gestion des ressources herbagères cultivées par des éleveurs laitiers sur un front pionnier en Amazonie brésilienne : cas du municipe de Uruar. Paris: INAPG.

Ingrand S, Astigarraga L, Chia E, David C, Coquil X, Fiorelli JL. 2009. Développer les propriétés de flexibilité des systèmes de production agricole en situation d'incertitude: pour une durabilité qui dure. $13^{\mathrm{e}}$ Journées de la recherche cunicole, 17-18 novembre 2009, Le Mans, France.

Lherm M, Veysset P, Bebin D. 2004. Évolutions constatées depuis 25 ans en exploitations d'élevage bovin charolais dans la zone herbagère nord Massif central. In: Colloque SFER, les systèmes de production agricole: performances, évolutions, perspectives. 18-19 novembre, Lille, France.

Rapey H. 2016. Les multiples formes actuelles de la conversion d'élevages laitiers de montagne vers la production de viande.
Journal of Alpine Research 104-4. Disponible sur http://rga. revues.org/3309 (mis en ligne le 18 juillet 2016, consulté le 04 janvier 2017). DOI : 10.4000/rga.3309.

Rouabhi A, Hafsi M, Monneveux P. 2018. Climate change during the last century in Setif province, Algeria. Agriculture 8(4): 60-75.

Rouget N. 2008. Les dynamiques agricoles dans les espaces urbains et périurbains. Diversification et stratégies d'adaptation des agricultures. Les cas des périphéries sud-est de Lille et nord de Lens. Thèse de géographie. Université de Nanterre Paris X. https:// halshs.archives-ouvertes.fr/tel-00477267/.

Ryschawy J, Choisis N, Choisis JP, Gibon A. 2013. Paths to last in mixed crop-livestock farming: lessons from an assessment of farm trajectories of change. Animal 7: 673-681.

Sraïri MT, Bahri S, Ghabiyel Y. 2018. Work management as a means to adapt to constraints in farming systems: a case study from two regions in Morocco. Cahiers agricultures 27: 15007. DOI: 10.1051 /cagri/2017066.

Tichit M, Hubert B, Doyen L, Genin D. 2004. A viability model to assess the sustainability of mixed herds under climatic uncertainty. Animal Research 53(5): 405-417. DOI: 10.1051/animres:2004024.

Citation de l'article : Semara L, Madani T, Mouffok C, Belkasmi F. 2018. Réaction des éleveurs bovins des régions semi-arides algériennes face aux contraintes économiques et climatiques. Cah. Agric. 27: 65001. 